\title{
Transductional Analysis of Arginineless Mutants in Proteus mirabilis
}

\author{
By O. W. PROZESKY \\ Department of Microbiology, University of Pretoria, Pretoria, South Africa
}

(Accepted for publication I3 June I968)

\begin{abstract}
SUMMARY
A genetic map of eight structural genes involved in arginine synthesis in Proteus mirabilis strain I 3 was obtained by transduction. Genes $\arg E, C, B$, $G, H$ form a cluster linked to the gene $c y s E$, the $\arg D$ gene is linked to a locus which controls resistance to $\mathrm{I} \mathrm{mg}$. $/ \mathrm{ml}$. streptomycin, $\arg F$ is linked to a pyrimidine marker, while the $\arg A$ gene could not be co-transduced with other markers. The clustering and order of $\arg E, C, B, H$, the linkage of $\arg D$ to str-r and the non-linkage of $\arg F$ and $\arg A$ to other arginine genes are features shared by $P$. mirabilis, Escherichia coli and Salmonella typhimurium. Proteus mirabilis differs from the other organisms in that arg $G$ is included in the $\arg E, C, B, G, H$ cluster. This cluster is closely linked to $c y s E$ and not to methionine markers. $A r g F$, which is the gene for the sixth step in the pathway, is linked to a pyrimidine marker while in $E$. coli this step is governed by two genes, one of which ( $\operatorname{argI})$ is linked to pyrB.
\end{abstract}

\section{INTRODUCTION}

In Proteus mirabilis strain 13 (Coetzee \& Sacks, 1960 b) the arginine pathway consists of eight enzymes which can be altered by single-step mutations (Prozesky, 1967). These enzymic steps are the same as those in Escherichia coli, and are outlined in Fig. I. To allow comparisons between gene maps the nomenclature is that of Glansdorff (1965, I967) and Prozesky (1967) but the equivalent designations used for Salmonella typhimurium (Sanderson \& Demerec, 1965) and strains of E. coli (Vogel, Bacon \& Baich, I963; Vogel \& Bacon, I966) are given in Fig. I. In E. coli K-12 the loci of seven structural arginine genes are distributed over four regions of the chromosome (Maas, I96I; Maas \& Maas, 1962). An eighth gene specific for step $D$ (Fig. I) has been identified in $E$. coli $\mathrm{w}$ and is situated in another region of the chromosome (Vogel, Bacon \& Baich, I963). An additional locus argI for the sixth step (Fig. I) was investigated by Glansdorff, Sand \& Verhoef (1967) in E. coli K-I2. They found $\operatorname{argI}$ situated separately from the other arg genes and linked to pyrB (Taylor \& Thoman, 1964). Masters \& Pardee (1965) found a mutation which results in a block at this step closely linked to pyrB in Bacillus subtilis.

The regulation of these scattered genes has been the subject of many investigations (see Baumberg, Bacon \& Vogel, 1965) and led to detailed examination of the $\arg E$, $C, B, H$ cluster in $E$. coli $\mathrm{K}-\mathrm{I} 2$ by Glansdorff $(1965,1967)$ and Sand \& Glansdorff (1967), who concluded that they could constitute an operon (Jacob, Perrin, Sanchez \& Monod, 1960). Other workers (Demerec et al. 1960; Sanderson \& Demerec, 1965; Armstrong, 1967) found the same arrangement of the corresponding genes in 
Salmonella typhimurium. Baumberg, Bacon \& Vogel (1966) found a pleiotropic mutation situated near this cluster which caused alterations in the regulation of genes $\arg C, B, H$ but not those of $\arg E$ or the unlinked $\arg D$ gene. They concluded that the $\arg E$ and $\arg H$ genes in the cluster are repressed separately although the $\arg E, C, B$, $H$ genes are closely linked. Sand \& Glansdorff (1967) described a polar $\arg E$ mutant which influences the enzyme levels of $\arg H$ in the cluster. They decided that the cluster could still be one operon.

The spatial arrangement of the eight structural genes of the arginine pathway of Proteus mirabilis was investigated in an attempt to resolve some aspects of this regulation problem as well as to contribute in general to genetic knowledge of Proteus mirabilis (Coetzee et al. 1966).

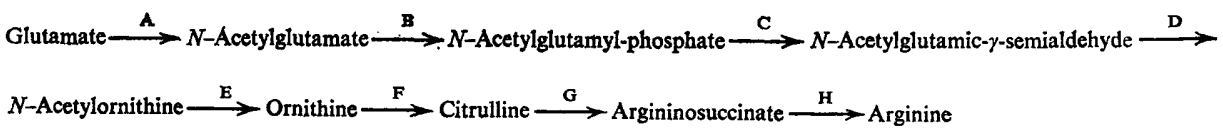

Fig. I. Pathway of arginine synthesis. A-H represent enzymic steps in Proteus mirabilis and Escherichia coli K-1 2 (Prozesky, 1967; Glansdorff, 1965) although step D mutants are only known in $E$. coli $\mathrm{w}$. The corresponding designations for these steps in the same sequence are B, C, H, G, A, D, E, F for E. coli w (Vogel \& Bacon, 1966) and Salmonella typhimurium (Sanderson \& Demerec, 1965).

\section{METHODS}

Media. The minimal medium was that used by Prozesky (1967). Growth factors were added to it (100 $\mu \mathrm{g} . / \mathrm{ml}$.) for selection of auxotrophic donor-type transductants: L-arginine hydrochloride, L-ornithine monohydrochloride, uracil, L-cysteine hydrochloride or DL-methionine (Nutritional Biochemicals Corporation, Cleveland, Ohio, U.S.A.). Difco SS agar or minimal agar containing I mg./ml. streptomycin sulphate was used for selection of streptomycin-resistant transductants. The broth was that of Coetzee \& Sacks (1960a).

Bacteria. Proteus mirabilis strain I3, a streptomycin-resistant mutant of this strain str-rI (the mutant I 3 str-r of Coetzee \& Sacks, 1960 $b$ ) and auxotrophic derivatives of these organisms were used (Table I). The biochemical characteristics of the arginineless mutants have been described (Prozesky, 1967). The two pyrimidine-requiring mutants pyr-3 and argF5pyr-2 have the same requirement for uracil as the mutant argF 3 pyr-Istr-r-I which was originally named $A C_{3} U r$ (Prozesky \& Coetzee, 1966). The blocks in the pyrimidine pathway of these mutants have not been investigated enzymically but their growth requirements and linkage by transduction to the $\arg F$ gene have been established (Prozesky \& Coetzee, 1966). The cysteineless and methionineless mutants were obtained from Mr W. O. K. Grabow (Grabow \& Smit, 1967). The cysE enzymic blocks of the selected double mutants argEIcysE5str-r-r and $\operatorname{argHIcys} E_{3}$ were also determined by these workers. Double arginineless mutants for three-point crosses were selected and characterized according to Prozesky \& Coetzee (1966) and Prozesky (1967). Streptomycin-resistant mutants resistant to I mg./ml. streptomycin were selected by the method of Coetzee \& Sacks (1960 b). Strains were maintained on agar slopes at $4^{\circ}$ and incubation temperature was $37^{\circ}$.

Transducing phage. The general phage techniques were those of Adams (I959). Lysates of phage 34/13 (Coetzee \& Sacks, 1960 $b$ ) with plaque-forming titres of $5 \times 10^{9}$ to $2 \times 10^{10}$ were prepared by an agar-layer technique (Prozesky, de Klerk 
Table I. Strains used for transduction analysis

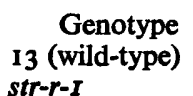

$s t r-r-I$

$\left.\begin{array}{ccc}\begin{array}{c}\text { Enzyme } \\ \text { blocks }\end{array} & \begin{array}{c}\text { Reaction } \\ \text { to strepto- } \\ \text { mycin }\end{array} & \\ \text { (Fig. I) } & \text { I mg./ml.* } & \begin{array}{c}\text { Phenotype } \\ \text { - }\end{array} \\ \hline- & \mathbf{s} & \begin{array}{l}\text { proto } \\ \text { proto }\end{array}\end{array}\right\}$

Arginineless mutants

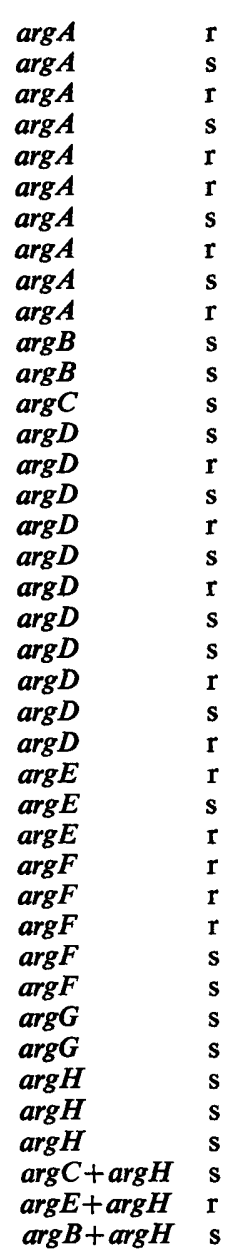

Growth response with:

$$
\begin{array}{r}
\text { orn, cit, arg } \\
\text { orn, cit, arg } \\
\text { orn, cit, arg } \\
\text { orn, cit, arg } \\
\text { orn, cit, arg } \\
\text { orn, cit, arg } \\
\text { orn, cit, arg } \\
\text { orn, cit, arg } \\
\text { orn, cit, arg } \\
\text { orn, cit, arg } \\
\text { orn, cit, arg } \\
\text { orn, cit, arg } \\
\text { orn, cit, arg } \\
\text { orn, cit, arg } \\
\text { orn, cit, arg } \\
\text { orn, cit, arg } \\
\text { orn, cit, arg } \\
\text { orn, cit, arg } \\
\text { orn, cit, arg } \\
\text { orn, cit, arg } \\
\text { orn, cit, arg } \\
\text { orn, cit, arg } \\
\text { orn, cit, arg } \\
\text { orn, cit, arg } \\
\text { orn, cit, arg } \\
\text { orn, cit, arg } \\
\text { orn, cit, arg } \\
\text { cit, arg } \\
\text { cit, arg } \\
\text { cit, arg } \\
\text { cit, arg } \\
\text { cit, arg } \\
\text { arg } \\
\text { arg } \\
\text { arg } \\
\text { arg } \\
\text { org }
\end{array}
$$

Prozesky, 1967
Reference
Coetzee \& Sacks,
I960 $b$

Assorted mutants

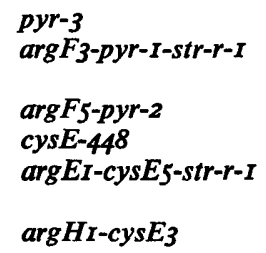

n.d.

$\arg F$ n.d. s

cysE

$\arg E+\operatorname{cys} E \quad \mathrm{r}$

$\arg H+\operatorname{cys} E \quad \mathrm{~s}$
Growth response with:

ura

arg, cit + ura

arg, cit + ura

hcys, cys, met

orn, cit, arg

+ hcys, cys, met

arg + hcys, cys, met
See Methods

Prozesky \& Coetzee,

See Methods

Grabow \& Smit, 1967

See Methods

See Methods

$* \mathrm{r}=$ resistant; $\mathrm{s}=$ sensitive; orn = ornithine; $\mathrm{cit}=$ citrulline; $\arg =$ arginine; ura $=$ uracil; hcys $=$ homocysteine $;$ cys $=$ cysteine $;$ met $=$ methionine; proto = prototroph; n.d. $=$ not done. 
\& Coetzee, 1965) on the wild-type organism and all mutants. Lysates were sterilized with chloroform and stored at $4^{\circ}$. Small numbers of phage I3vir (Prozesky et al. 1965) were sometimes present in transducing lysates. The effect of these virulent phages on the transduction frequency was tested in reconstruction experiments as follows: Phage I3vir was prepared by the method of Prozesky et al. (1965). Dilutions of the I 3 vir lysates were made in a lysate of transducing phage $34 / 13$ and samples of the mixed lysates were then used to transduce two auxotrophs to prototrophy.

Transduction. This procedure has been described (Prozesky \& Coetzee, 1966). Phage lysate ( $\mathrm{I} \mathrm{ml}$.) was mixed with I $\mathrm{ml}$. of a $16 \mathrm{hr}$ broth culture of recipient bacteria (about $2 \times 10^{\circ} / \mathrm{ml}$.) and incubated without shaking. After adsorption for $15 \mathrm{~min}$. the bacteria were spun down, the pellets resuspended in $0.9 \%(\mathrm{w} / \mathrm{v}) \mathrm{NaCl}$ and various dilutions plated. Phage sterility controls as well as controls in which recipients were treated with lysates prepared on homologous and wild-type bacteria respectively were included. Results were read after $48 \mathrm{hr}$.

Selection of transductants. Wild-type transductants were selected on minimal medium and auxotrophic donor-type transductants on appropriately supplemented minimal medium (Lennox, 1955). Plates with donor-type transductants were replicated to minimal medium for scoring (Lederberg \& Lederberg, 1952) and read after $24 \mathrm{hr}$. When streptomycin resistance was the unselected marker, prototroph colonies on minimal medium were replicated to minimal medium with streptomycin. In crosses between $\arg G$ or $\arg H$ recipients and $\arg B, C$ or $E$ mutant class donors the master plate technique (Glansdorff, I965) was used to obviate feeding of the donor-type recombinant classes by the background growth of the recipient (Prozesky, 1967).

Linked transduction. Linked transduction of arginine genes with other markers was demonstrated by the use of arginineless mutants as donors with various mutant recipients and selection for arg-donor-type transductants (Demerec et al. 1956). Non-replicating colonies were picked off into broth, purified and tested for inheritance of the donor-type by auxanography. Results which suggested linked transduction were confirmed by reverse crosses with selection for the opposite donor-type.

Genetic mapping. Two-point transductions were performed by crosses of single mutants in all combinations. The number of colonies on control plates of recipients treated with homologous phage were subtracted from those on test plates. Three-point linkage tests were done with the mutants in seven of the genes where linkage to external markers was found. In three-point experiments double mutants with linked markers were crossed with single mutants for the determination of the order of the genes and the mutations order by donor-switching (Glansdorff, 1965). The crossovers involved in the formation of wild-type transductants in the respective crosses have been discussed and illustrated by Clowes (1960) and Glansdorff (1965). The sequence of the three markers involved in the crosses can be derived from the ratio of the number of transductants from a cross to the number obtained from the reverse cross. A low ratio is indicative of an unequal number of crossovers while a ratio of about unity means that the same number of crossovers are involved in the two crosses. A similar method with selection for $\mathrm{arg}^{+}, s t r-r$ transductants was used to map the $\arg D$ mutations with $s t r-r$ as the linked marker. Relative map distances were determined by a ratio test with a linked marker (Lennox, 1955; Gross \& Englesberg, 1959). This ratio is expressed as 'wild-type transductants per total transductants' in per cent or with the $\arg D$ and str-r markers as 'str-, arg- transductants per str-r transductants' in per cent. 
The $\arg A$ mutants were mapped by a ratio test with the unlinked str-r marker. Streptomycin-resistant mutants were selected from each of the $\arg A$ mutants and crossed with the other streptomycin-sensitive mutants in the gene. Selection was made separately for prototrophy and streptomycin-resistance. The number of prototrophs was then compared with the number of streptomycin-resistant transductants from the same cross.

Purity of transductant clones. This was checked in two crosses: $50 \mathrm{arg}^{+}$colonies from each of the transductions $\arg E_{\mathrm{I}} c_{\text {cys }} E_{5 s t r-r-I} \times \arg H_{I}$ and $\arg H_{I}{ }_{c y s} E_{3} \times \arg E_{1}$ were tested for the cys $E$ marker by auxanography.

\section{RESULTS}

Mutants. The streptomycin-resistant mutants are resistant to $\mathrm{I} \mathrm{mg} . / \mathrm{ml}$., exhibit no additional auxotrophy (Sanderson $\&$ Demerec, 1965) and are linked to the $\arg D$ gene (see below). These mutants correspond to the str- $A$ mutants of Sanderson \& Demerec (1965). The co-transduction findings (Table Io) indicate that the streptomycin mutants are situated in a single locus. The auxotrophic mutants were obtained with the use of $\mathrm{MnCl}_{2}$ (Prozesky, 1967) and behave like revertable point mutations except for mutant $\arg D_{4}$ which may be a multisite mutant. No true deletions (Hartman, Loper \& Serman, I960) were encountered.

Table 2. Effect of phage I 3 vir on transduction frequency

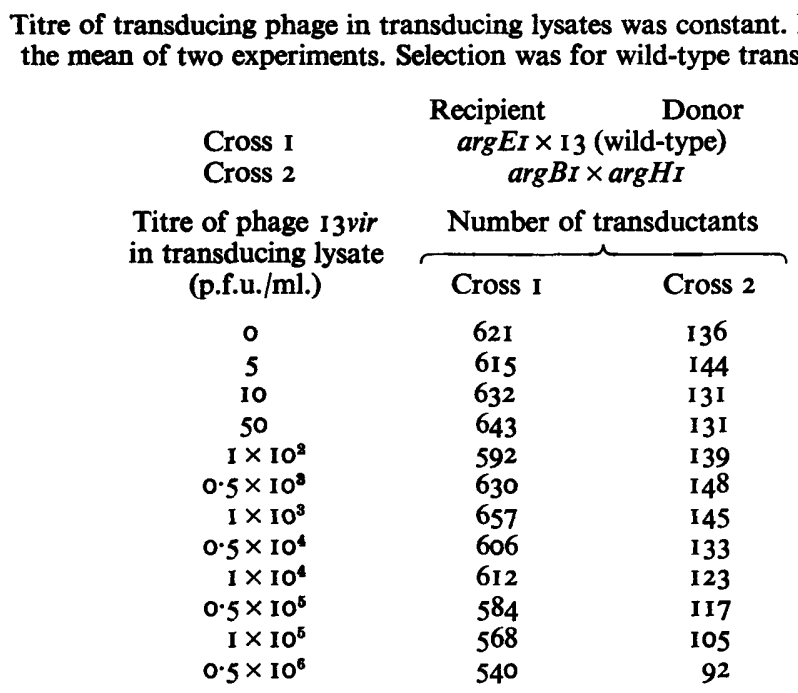

The effect of virulent phage on the transduction frequency. There was a significant decrease in the yield of transductants (Table 2) when transducing lysates contained more than $\mathrm{I} \times 10^{4}$ plaque-forming units of phage $13 \mathrm{vir}$ per $/ \mathrm{ml}$. Lysates used in this investigation never contained more than 50 plaque-forming units (p.f.u.) of phage 13vir/per ml.

Purity of transductant clones. No mixed clones were encountered and the incidence of such colonies was taken to be negligible (Glansdorff, 1965). 
Linkage of other markers to arginine genes. Linked transduction of an $\arg F$ and a pyrimidine marker has been described (Prozesky \& Coetzee, I966). In crosses between arginineless mutants and representative cysteineless and methionineless mutants of Grabow \& Smit (1967) donor-type transduction occurred only with the cysE and $\arg B, C, E, G$ and $H$ markers. Streptomycin resistance could only be co-transduced with the $\arg D$ marker. The $\arg A$ mutants are not linked to any other marker (also see Prozesky \& Coetzee, 1966). Seven of the eight arginine genes could thus be mapped with linked markers.

Two-point crosses for preliminary mapping. Results of an experiment are given in Table 3. Small numbers of transductants were obtained from intragenic crosses or crosses between sites in closely linked genes. Mutants $\operatorname{argF} 4$ and $\operatorname{argHz}$ whether used as recipients or donors always yielded larger numbers of transductants than other mutants. Mutants $\operatorname{argAI}$ and $\arg D_{4}$ on the other hand were poor recipients with average donor capacities. The efficiency of plating of phage $34 / 13$ on these four mutants is unity. Mutant $\operatorname{argF} 4$ is slightly 'leaky' but has a low spontaneous reversion rate (Table 3). Numbers of colonies on control plates which form a column diagonally across Table 3 show that the reversion rate of the mutants is low except in the case of $\operatorname{argG2}$. This mutant was retained in the series as it and $\arg G_{I}$ are the only representatives of their class. The bold figures in Table 3 delineate genes and lines indicate linked genes. Indications of gene arrangement were obtained from these findings. The $\arg B$ and $\arg C$ mutants yield very small numbers of transductants in reciprocal crosses and were taken to be closely linked. Crosses between $\arg E$ and $\arg H$ mutants give rise to more transductants than other members of the $\arg B, C, E, G, H$ group and were expected to be at the extremities of the cluster.

Three-point linkage tests with the arg $B, C, E, G, H$ mutants. Double mutants with known $\arg B, C$ or $E$ mutations and additional $\arg H$ mutations (Table I) were used in reciprocal experiments with single $\arg B, C, E, G$ or $H$ mutants. Results are given in Table 4. The ratios of the numbers of transductants obtained in the reciprocal crosses fall into two groups, namely $0-0.2$ and $0.6-\mathrm{I} \cdot 3$. The gene order established is $\arg E-C-$ $B-G-H$ and the relative positions of some of the mutants are

$$
\begin{aligned}
& \arg \mathrm{EI}_{\mathrm{I}}-\mathrm{E}_{2}-\mathrm{CI}_{\mathrm{I}} \mathrm{BI}-\mathrm{B2}_{2}-\mathrm{GI}_{\mathrm{I}} \mathrm{H}_{5}-\mathrm{H}_{2}-\mathrm{H}_{4}-\mathrm{HI}_{\mathrm{I}} \text {. } \\
& \left(\begin{array}{lll}
\left.E_{3}\right) & \left(\mathrm{G}_{2}\right) & \left(\mathrm{H}_{3}\right)\left(\mathrm{H}_{6}\right)
\end{array}\right.
\end{aligned}
$$

Mutants in parentheses are assigned to the same positions occupied by those above them.

Three-point linkage tests with the argE, $C, B, G, H$ mutants and external cysE markers. Two double mutants, argErcysE5 and $\operatorname{argHrcys} E_{3}$, were used in donor-switching experiments. Results of a typical experiment are given in Table 5. One group of ratios ranged from 0.5 to 0.8 and the other from 0 to 0.2 . The mutant order above was confirmed in that all the single arg mutations as well as cysE448 mapped between $c y s E_{3}$ and $\arg H I$ and all the mutations except cysE448 mapped to the 'right' of $\operatorname{argEI}$ and cysE5. The final map is given in Fig. 2.

Relative distances within the $\arg E, C, B, G, H$ gene cluster. The results of the ratio test with ornithine-requiring $\arg E, C$ or $B$ donors and arginine-requiring $\arg G$ or $\arg H$ recipients (Glansdorff, 1965) are given in Table 6. Results confirm the marker order arrived at above and also indicate the positions of mutants which could not be placed with certainty. By this test the position of $\operatorname{argHz}$ seems to be farther to the 
Table 4. Three-point transductions with the $\arg B, C, E, G, H$ mutants

\begin{tabular}{|c|c|c|c|}
\hline Cross & \multirow{2}{*}{$\begin{array}{l}\text { Prototroph } \\
\text { transductants } \\
\text { obtained }\end{array}$} & \multirow{2}{*}{$\begin{array}{c}\text { Ratio } \\
a \mid b\end{array}$} & \multirow[b]{2}{*}{ Mutant order } \\
\hline Recipient & & & \\
\hline (a) $\operatorname{argBIargH} 6 \times \operatorname{argEI}$ & 8 & $1 \cdot 3$ & $\operatorname{argEI} \operatorname{argBI} \arg H 6$ \\
\hline (b) argEI $\times$ argBIargH6 & 6 & & \\
\hline (a) $\operatorname{argB1argH6} \times$ argE2 & 4 & 0.8 & $\operatorname{argE2} \operatorname{argBI} \arg H 6$ \\
\hline (b) $\operatorname{argE2} 2 \times \operatorname{argBiargH6}$ & 5 & & \\
\hline (a) $\operatorname{argBIargH} 6 \times \arg E_{3}$ & I I & $\mathbf{I} \cdot \mathbf{I}$ & $\arg E_{3} \arg B I \arg H 6$ \\
\hline (b) $\arg E_{3} \quad \times$ argBIargH6 & 10 & & \\
\hline (a) $\operatorname{argBIarg} \mathrm{H} 6 \times \operatorname{argCI}$ & 2 & 0.7 & $\operatorname{argCI} \operatorname{argBI} \operatorname{argH} 6$ \\
\hline (b) $\operatorname{argCI} \times$ argBrargH6 & 3 & & \\
\hline (a) argBIarg $H 6 \times$ argBI & 0 & - & - \\
\hline (b) argBI $\times$ argBIargH6 & 0 & & \\
\hline (a) argBIargH $6 \times$ argB2 & 0 & o & $\operatorname{argBr} \operatorname{argB2} \operatorname{argH} 6$ \\
\hline (b) $\operatorname{argB2} \times$ argBIargH6 & 2 & & \\
\hline (a) argBIarg $H 6 \times$ argGI & I & 0.1 & $\operatorname{argBI} \operatorname{argGI} \operatorname{argH6}$ \\
\hline (b) argGI $\times$ argBIargH6 & 23 & & \\
\hline (a) argBIarg $H 6 \times$ argG2 & 4 & 0.1 & $\operatorname{argBI} \operatorname{argG2} \operatorname{argH6}$ \\
\hline (b) $\operatorname{argG2} \times$ argBiargH6 & 46 & & \\
\hline (a) $\operatorname{argBiarg} H 6 \times$ argHI & 6 & $1 \cdot 5$ & $\operatorname{argBI} \operatorname{argH6} \operatorname{argHI}$ \\
\hline (b) $\operatorname{argHI} \quad \times$ argBIargH6 & 4 & & \\
\hline (a) $\operatorname{argB1}$ arg $\mathrm{H} 6 \times$ argH2 & i & 0.1 & $\operatorname{argBI} \operatorname{argH2} \operatorname{argH} 6$ \\
\hline (b) $\operatorname{argH2} \times$ argBIargH6 & 17 & & \\
\hline (a) $\operatorname{argBiargH} 6 \times \arg \mathrm{H}_{3}$ & 0 & 0 & $\operatorname{argBI} \operatorname{argH} 3 \operatorname{argH} 6$ \\
\hline (b) $\operatorname{argH} 33$ & 5 & & \\
\hline (a) $\operatorname{argEIarg} \mathrm{H}_{5} \times \operatorname{argEI}$ & 0 & - & - \\
\hline (b) $\operatorname{argEI} \times$ argEIarg $H_{5}$ & 0 & & \\
\hline (a) $\operatorname{argErarg} H_{5} \times \operatorname{argE2}$ & 0 & 0 & $\operatorname{argEI} \operatorname{argE2} 2 \arg H_{5}$ \\
\hline (b) argE2 $\times$ argErargH5 & II & & \\
\hline (a) $\arg E_{1} a r g H_{5} \times \arg E_{3}$ & 0 & o & $\arg E_{1} \arg E_{3} \arg H_{5}$ \\
\hline (b) $\arg E_{3} \times$ argErargH 5 & 2 & & \\
\hline (a) $\operatorname{argEIarg} \mathrm{H}_{5} \times \arg \mathrm{CI}_{\mathrm{I}}$ & 2 & 0.1 & $\operatorname{argEI} \arg \mathrm{CI}_{\mathrm{I}} \arg \mathrm{H}_{5}$ \\
\hline (b) $\operatorname{argCI} \times$ argEIargH5 & 20 & & \\
\hline (a) argEIarg $H_{5} \times$ argBI & I & O.I & $\arg E_{I} \operatorname{argBI} \arg \mathrm{H}_{5}$ \\
\hline (b) $\operatorname{argBI} \times \operatorname{argEIarg} H_{5}$ & 12 & & \\
\hline (a) $\operatorname{argE} \operatorname{Iarg} \mathrm{H}_{5} \times \operatorname{argB2}$ & 3 & 0.2 & $\operatorname{argEI} \operatorname{argB2} \arg H_{5}$ \\
\hline (b) argB2 $\times$ argErargH5 & 17 & & \\
\hline (a) $\arg E_{1} \arg H_{5} \times$ argGr & 4 & $0 \cdot \mathbf{I}$ & $\arg E_{I} \operatorname{argGr} \arg \mathrm{H}_{5}$ \\
\hline (b) argGr $\times$ argErarg $H_{5}$ & 73 & & \\
\hline (a) $\arg E_{1} a r g H_{5} \times$ argG2 & 2 & 0.1 & $\operatorname{argEI} \operatorname{argG} 2 \arg H_{5}$ \\
\hline (b) argG2 $\times$ argErargH5 & $3 \mathrm{I}$ & & \\
\hline (a) $\operatorname{argEIarg} H_{5} \times \operatorname{argHI}$ & 3 & 0.8 & $\operatorname{argEI} \operatorname{argH} 5 \operatorname{argHI}$ \\
\hline (b) argHI $\times$ argEIargH 5 & 4 & & \\
\hline (a) argE1arg $H_{5} \times$ arg $H_{2}$ & 32 & 0.6 & $\operatorname{argEI} \arg H_{5} \operatorname{argHz}$ \\
\hline (b) $\operatorname{argH} 2 \quad \times$ argEIargH 5 & 53 & & \\
\hline (a) $\operatorname{argEIarg} \mathrm{H}_{5} \times \mathrm{argH}_{3}$ & $\begin{array}{l}4 \\
6\end{array}$ & $0 \cdot 7$ & $\operatorname{argEI} \operatorname{argH} 5$ arg $H_{3}$ \\
\hline $\begin{array}{l}\text { (b) } \operatorname{argH} 3 \times \text { argEIargH5 } \\
\text { (a) } \operatorname{argClargH} 4 \times \operatorname{argEI}\end{array}$ & $\begin{array}{r}6 \\
\text { II }\end{array}$ & 0.7 & $\operatorname{argEr} \operatorname{argCr} \operatorname{argH}_{4}$ \\
\hline (b) $\operatorname{argEI} \times \operatorname{argCIargH}_{4}$ & 16 & & \\
\hline (a) $\arg$ Irarg $_{4} \times$ argE2 & 24 & 0.8 & $\operatorname{argE2} \operatorname{argCI} \operatorname{argH} 4$ \\
\hline (b) $\operatorname{argE2} \times$ argCrargH 4 & $3 \mathrm{I}$ & & \\
\hline (a) $\operatorname{argCrarg} \mathrm{H}_{4} \times \operatorname{argE} E_{3}$ & 23 & 0.7 & $\arg E_{3} \arg \mathrm{CI}_{\mathrm{I}} \operatorname{argH} 4$ \\
\hline (b) $\operatorname{argE}_{3} \times \operatorname{argCrargH} 4$ & 35 & & \\
\hline (a) $\operatorname{argCrarg} \mathrm{H}_{4} \times \operatorname{argCI}$ & 0 & - & - \\
\hline (b) $\operatorname{argCI} \times \operatorname{argCIargH} 4$ & o & & \\
\hline (a) $\operatorname{argCrarg} H_{4} \times \operatorname{argBI}$ & 0 & 0 & $\operatorname{argCr} \operatorname{argBr} \arg H_{4}$ \\
\hline (b) $\operatorname{argBI} \times \operatorname{argCrargH} 4$ & 4 & & \\
\hline (a) $\operatorname{argCIarg} \mathrm{H}_{4} \times \operatorname{argB2}$ & I & $0 \cdot I$ & $\operatorname{argCr} \operatorname{argB2} \operatorname{argH}_{4}$ \\
\hline (b) $\operatorname{argB2} \times \operatorname{argCrargH}_{4}$ & 9 & & \\
\hline (a) $\arg \mathrm{Crarg}_{4} \times$ argGI & 2 & $0 \cdot I$ & $\operatorname{argCr} \operatorname{argGI} \operatorname{argH} 4$ \\
\hline (b) $\operatorname{argGI} \times \operatorname{argCrarg} \mathrm{H}_{4}$ & 18 & & \\
\hline (a) $\operatorname{argCrarg} \mathrm{H}_{4} \times$ argG2 & 3 & O.I & $\arg \mathrm{CI}_{1} \operatorname{argG} 2 \arg \mathrm{H}_{4}$ \\
\hline (b) $\operatorname{argG2} \times \operatorname{argCIargH}_{4}$ & 24 & & \\
\hline (a) argCrarg $\mathrm{H}_{4} \times$ argHI & 3 & 0.6 & $\operatorname{argCr} \operatorname{argH} 4 \operatorname{argHr}$ \\
\hline (b) $\operatorname{argHI} \times \operatorname{argCrargH} 4$ & 5 & & \\
\hline (a) $\operatorname{argCrarg} \mathrm{H}_{4} \times \operatorname{argH}_{2}$ & 0 & 0 & $\operatorname{argCI} \operatorname{argH} 2 \operatorname{argH} 4$ \\
\hline (b) $\underset{\mathrm{argH}}{ } \times \operatorname{argCrargH}_{4}$ & 3 & & \\
\hline (a) $\operatorname{argCIargH} 4 \times \operatorname{argH}_{3}$ & 0 & 0 & $\arg \mathrm{Cl}_{\mathrm{I}} \arg \mathrm{H}_{3} \arg \mathrm{H}_{4}$ \\
\hline (b) arg Ha $\times$ argCrargH4 & 7 & & \\
\hline
\end{tabular}


'right' than indicated by the previous test. Like the Escherichia coli mutant $\operatorname{argC2}$ (Glansdorff, 1965), argHz yields more wild-type transductants than other mutants and can only be accurately mapped by three-point transductions which correct for the high rate of transduction to prototrophy.

Relative distances of the arg $E, C, B, G, H$ mutations from the linked external marker cysE448. These results are presented in Table 7 and confirm the order derived above. Map distances appear slightly smaller than in the previous ratio test (Table 6). This

Table 5. Three-point transductions with the argE, $C, B, G, H$ mutants and the external marker cysE

\begin{tabular}{|c|c|c|}
\hline \multicolumn{3}{|c|}{ Cross } \\
\hline & Recipient & Donor \\
\hline (a) & $\operatorname{argErcys} E$ & $5 \times$ cysE 448 \\
\hline (b) & cysE 448 & $\times$ argEIcysE5 \\
\hline (a) & $\operatorname{argErcys} E$ & $5 \times \arg E_{I}$ \\
\hline (b) & $\arg E I$ & $\times$ argEIcysEs \\
\hline (a) & $\operatorname{argEIcys} E$ & $5 \times \operatorname{argE2}$ \\
\hline (b) & $\operatorname{argE2}$ & $\times \operatorname{argEIcys}{ }_{5}$ \\
\hline (a) & $\operatorname{argEIcys} E$ & $5 \times \arg E_{3}$ \\
\hline (b) & $\arg E_{3}$ & $\times \arg E_{1} c y s E_{5}$ \\
\hline (a) & $\operatorname{argEIcys} E$ & $5 \times \arg C I$ \\
\hline (b) & $\arg C I$ & $\times \arg E_{1} c y s E_{5}$ \\
\hline (a) & $\operatorname{argEIcys} E$ & $5 \times \operatorname{argBI}$ \\
\hline (b) & $\arg B I$ & $\times$ argEIcysE5 \\
\hline (a) & $\arg E_{I c y s} E$ & $5 \times \operatorname{argB2}$ \\
\hline (b) & $\arg B 2$ & $\times$ argErcysE5 \\
\hline (a) & $\arg E I c y_{s} E$ & $5 \times \arg G I$ \\
\hline (b) & $\operatorname{argGI}$ & $\times \arg E_{1 c y s} E_{5}$ \\
\hline (a) & $\operatorname{argEIcys} E$ & $5 \times \operatorname{argG2}$ \\
\hline (b) & $\operatorname{argG2}$ & $\times$ argEIcysE5 \\
\hline (a) & $\operatorname{argEIcys}$ & $5 \times \operatorname{argHI}$ \\
\hline (b) & $\operatorname{argHI}$ & $\times$ argEIcysEs \\
\hline (a) & $\operatorname{argEIcys} E$ & $5 \times \operatorname{argHz}$ \\
\hline $\begin{array}{l}\text { (b) } \\
\text { (a) }\end{array}$ & $\begin{array}{l}\operatorname{argHz} \\
\operatorname{argEIC}\end{array}$ & $\times$ argEIcysE5 \\
\hline $\begin{array}{l}\text { (a) } \\
\text { (b) }\end{array}$ & $\begin{array}{l}\operatorname{argEIcy} \\
\operatorname{argH}\end{array}$ & $\begin{array}{l}5 \times \operatorname{argH} 3 \\
\times \operatorname{argEICys} E_{5}\end{array}$ \\
\hline (a) & argHicys & $3 \times$ cys $_{448}$ \\
\hline (b) & cys $E 448$ & $\times$ argHICysE 3 \\
\hline (a) & $\operatorname{argHICys} E$ & $3 \times \operatorname{argEr}$ \\
\hline (b) & $\arg E I$ & $\times$ argHicysE3 \\
\hline (a) & $\operatorname{argHIcys}$ & $3 \times \operatorname{argE2}$ \\
\hline (b) & $\operatorname{argE2}$ & $\times \operatorname{argHIcysE_{3}}$ \\
\hline (a) & $\operatorname{argHicys} E$ & $3 \times \arg E_{3}$ \\
\hline (b) & $\arg E_{3}$ & $\times$ argHIcysE3 \\
\hline (a) & $\operatorname{argHIcysE}$ & $3 \times \arg C I$ \\
\hline (b) & $\operatorname{argCI}$ & $\times$ argHIcysE3 \\
\hline (a) & $\operatorname{argHrcysE}$ & $3 \times \operatorname{argB2}$ \\
\hline (b) & $\operatorname{argB2}$ & $\times$ argHIcysE 3 \\
\hline (a) & $\operatorname{argHIcys}$ & $3 \times \operatorname{argGI}$ \\
\hline (b) & $\arg G I$ & $\times \operatorname{argHIcys} E_{3}$ \\
\hline (a) & $\operatorname{argHIcys} E$ & $3 \times \operatorname{argHI}$ \\
\hline (b) & $\operatorname{argHI}$ & $\times$ argHIcysE3 \\
\hline (a) & $\operatorname{argHIcysE}$ & $3 \times \operatorname{argH} 2$ \\
\hline (b) & $\operatorname{argH} 2$ & $\times \operatorname{argHI}$ cysE 3 \\
\hline (a) & $\operatorname{argHIcys}$ & $3 \times \operatorname{argn3}$ \\
\hline (b) & $\arg H_{3}$ & $\times$ argHIcysE3 \\
\hline
\end{tabular}

Prototroph transductants obtained

Ratio

O. I

3
66

0

0

2

3

4

I 1

I 6

16

20

IO

16

I 85

302

152

212

64

120

180

304

96

184

0

21

3

54

3

43

8

37

2

34

0

29

5
85

0

0
17

7

0.7

0.5

0.7

0.8

0.6

0.6

0.7

0.5

0.6

0.5

o

0.1

O. I

0.2

O. I

o

O. I

0

o
Mutant order

cysE5 cysE448 argEI $\operatorname{cys}_{3} \operatorname{argHz} \operatorname{argHI}$

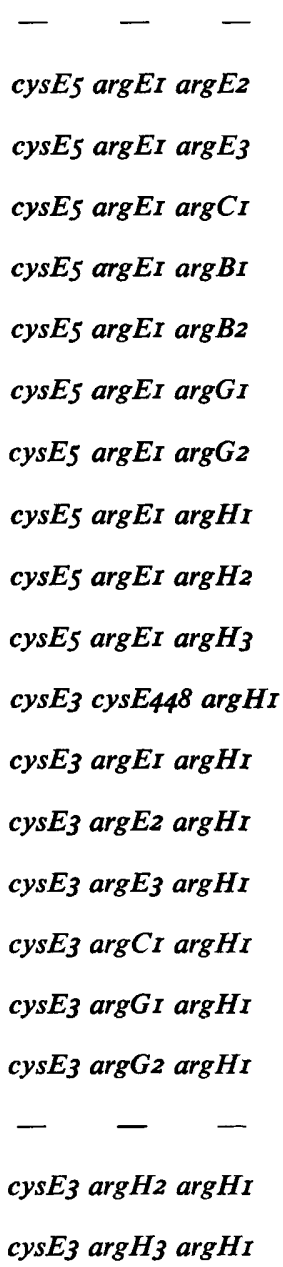


Table 6. Distances between $\arg E, C, B$, and arg $G$ and $H$ mutants

Values are the mean of two experiments. Mutant argG2 was not used because of its high reversion rate.

\begin{tabular}{|c|c|c|}
\hline Cross & \multirow{2}{*}{$\begin{array}{l}\text { Transductants: } \\
\text { prototrophs/total }\end{array}$} & \multirow[b]{2}{*}{$\%$} \\
\hline Recipient & & \\
\hline $\arg G I \times \operatorname{argEI}$ & $83 / 779$ & $10 \cdot 7$ \\
\hline $\operatorname{argGI} \times \arg E_{3}$ & $91 / 900$ & $9 \cdot 9$ \\
\hline $\arg G I \times \operatorname{argE2}$ & $60 / 854$ & \\
\hline $\arg G I \times \arg C I$ & $62 / 1150$ & $5 \cdot 4$ \\
\hline $\arg G I \times \arg B I$ & $59 / 1$ I 84 & 5.0 \\
\hline $\operatorname{argGI} \times \arg B 2$ & $31 / 803$ & $2 \cdot 6$ \\
\hline $\arg H_{3} \times \operatorname{argEI}$ & $82 / 481$ & $19 \cdot 1$ \\
\hline $\arg H_{3} \times \arg E_{3}$ & $211 / 1148$ & $18 \cdot 4$ \\
\hline $\operatorname{argH} H_{3} \times \operatorname{argE2}$ & $128 / 810$ & $15 \cdot 8$ \\
\hline $\operatorname{argH} 3 \times \operatorname{argCI}$ & $177 / 1308$ & 13.5 \\
\hline $\arg H_{3} \times \operatorname{argBr}$ & I 27/975 & 13 \\
\hline $\operatorname{argH} 3 \times \operatorname{argBz}$ & $68 / 63 I$ & 10.8 \\
\hline $\operatorname{argHz} \times \arg E_{I}$ & $412 / 970$ & 42 \\
\hline $\arg H_{2} \times \arg E_{3}$ & $811 / 2102$ & 38 \\
\hline $\operatorname{argH2} \times \operatorname{argE2}$ & $734 / 2331$ & $32 \cdot 4$ \\
\hline $\operatorname{argH} 2 \times \arg C I$ & $318 / 1112$ & $28 \cdot 7$ \\
\hline $\arg H_{2} \times \operatorname{argBI}$ & $542 / 1993$ & $27 \cdot 2$ \\
\hline $\operatorname{argH2} \times \operatorname{argB2}$ & $228 / 1155$ & 19.7 \\
\hline $\operatorname{argHI} \times \operatorname{argEI}$ & $160 / 828$ & $19 \cdot 3$ \\
\hline $\operatorname{argHI} \times \operatorname{argE} 3$ & $163 / 878$ & $18 \cdot 6$ \\
\hline $\operatorname{argHI} \times \operatorname{argE2}$ & I 27/802 & $15 \cdot 8$ \\
\hline $\operatorname{argHI} \times \arg C I$ & I $16 / 847$ & $13 \cdot 7$ \\
\hline $\arg H I \times \arg B I$ & $126 / 960$ & $13 \cdot 1$ \\
\hline $\arg H I \times \operatorname{argB2}$ & $64 / 611$ & $10 \cdot 6$ \\
\hline
\end{tabular}

Table 7. Distances between argE, $C, B, G, H$ mutants and the cysE448 marker Values are the mean of two experiments.

\begin{tabular}{|c|c|c|c|c|}
\hline \multicolumn{2}{|c|}{ Cross } & \multirow{2}{*}{$\begin{array}{l}\text { Transductants: } \\
\text { prototrophs/total }\end{array}$} & \multirow[b]{2}{*}{$\%$} & \multirow[b]{2}{*}{ Average } \\
\hline Recipient & Donor & & & \\
\hline $\begin{array}{l}\arg E I \\
\text { cysE } 448\end{array}$ & $\begin{array}{l}\times \operatorname{cys} E 448 \\
8 \times \operatorname{argEI}\end{array}$ & $\begin{array}{l}186 / 238 \\
143 / 186\end{array}$ & $\left.\begin{array}{l}78 \cdot 2 \\
76 \cdot 9\end{array}\right\}$ & $77 \cdot 6$ \\
\hline $\begin{array}{l}\arg E_{3} \\
\text { cys } E_{448}\end{array}$ & $\begin{array}{l}\times \operatorname{cys} E_{448} \\
8 \times \arg E_{3}\end{array}$ & $\begin{array}{l}196 / 246 \\
190 / 248\end{array}$ & $\left.\begin{array}{l}79 \cdot 7 \\
76 \cdot 6\end{array}\right\}$ & $78 \cdot 2$ \\
\hline $\begin{array}{l}\arg E_{2} \\
\text { cysE448 }\end{array}$ & $\begin{array}{l}\times \operatorname{cys} E_{448} \\
8 \times \arg E_{2}\end{array}$ & $\begin{array}{l}208 / 258 \\
156 / 196\end{array}$ & $\left.\begin{array}{l}80 \cdot 6 \\
79 \cdot 6\end{array}\right\}$ & $80 \cdot 1$ \\
\hline $\begin{array}{l}\arg C I \\
\text { cysE } E 44^{8}\end{array}$ & $\begin{array}{l}\times \text { cysE } 448 \\
8 \times \operatorname{argCI}\end{array}$ & $\begin{array}{l}252 / 307 \\
366 / 452\end{array}$ & $\left.\begin{array}{l}82 \cdot I \\
8 I \cdot 0\end{array}\right\}$ & $8 I \cdot 6$ \\
\hline $\begin{array}{l}\arg B I \\
\text { cysE } E 44^{8}\end{array}$ & $\begin{array}{l}\times \operatorname{cysE} 448 \\
8 \times \operatorname{argBI}\end{array}$ & $\begin{array}{l}183 / 228 \\
188 / 224\end{array}$ & $\left.\begin{array}{l}80.3 \\
83.9\end{array}\right\}$ & $82 \cdot 1$ \\
\hline $\begin{array}{l}\operatorname{argB2} \\
\text { cysE } 448\end{array}$ & $\begin{array}{l}\times \text { cysE } 448 \\
8 \times \operatorname{argBz}\end{array}$ & $\begin{array}{l}289 / 350 \\
260 / 307\end{array}$ & $\left.\begin{array}{l}82 \cdot 6 \\
84 \cdot 7\end{array}\right\}$ & $83 \cdot 7$ \\
\hline $\begin{array}{l}\arg G_{2} \\
\text { cysE } E_{448}\end{array}$ & $\begin{array}{l}\times \text { cysE } 448 \\
8 \times \arg G 2\end{array}$ & $\begin{array}{l}395 / 452 \\
192 / 234\end{array}$ & $\left.\begin{array}{l}87.4 \\
82.1\end{array}\right\}$ & $84 \cdot 8$ \\
\hline $\begin{array}{l}\arg G I \\
\text { cysE448 }\end{array}$ & $\begin{array}{l}\times \operatorname{cys} E 448 \\
8 \times \arg G I\end{array}$ & $\begin{array}{l}101 / 117 \\
265 / 299\end{array}$ & $\left.\begin{array}{l}86 \cdot 3 \\
88 \cdot 6\end{array}\right\}$ & $87 \cdot 5$ \\
\hline $\begin{array}{l}\operatorname{argH2} \\
\operatorname{cys} E_{448}\end{array}$ & $\begin{array}{l}\times \text { cysE448 } \\
8 \times \text { argH2 }\end{array}$ & $\begin{array}{l}452 / 482 \\
378 / 402\end{array}$ & $\left.\begin{array}{l}93 \cdot 8 \\
94 \cdot 0\end{array}\right\}$ & $93 \cdot 9$ \\
\hline $\begin{array}{l}\underset{\text { cys }}{\arg H_{3}} \\
44^{8}\end{array}$ & $\begin{array}{l}\times \text { cysE }{ }_{448} \\
8 \times \text { arg H3 }\end{array}$ & $\begin{array}{l}219 / 256 \\
358 / 386\end{array}$ & $\left.\begin{array}{l}85 \cdot 6 \\
92 \cdot 8\end{array}\right\}$ & $89 \cdot 2$ \\
\hline $\begin{array}{l}\operatorname{argHI} \\
\text { cysE448 }\end{array}$ & $\begin{array}{l}\times \text { cysE448 } \\
8 \times \operatorname{argHI}\end{array}$ & $\begin{array}{l}339 / 368 \\
244 / 268\end{array}$ & $\left.\begin{array}{l}92 \cdot 1 \\
91 \cdot 0\end{array}\right\}$ & $9 I \cdot 6$ \\
\hline
\end{tabular}


discrepancy is possibly caused by different efficiencies of pairing in different regions of the chromosome (Pritchard, 1960).

Donor-switching experiments with the arg $F$ and pyr markers. Two double mutants $\operatorname{argF} 3 p y r-I$ and $\arg F_{5 p y r-2}$ were crossed with the single $\arg F$ and $p y r-3$ mutants. Results (Table 8) indicate that pyr-Ipyr-2 and pyr-3 are situated on the same side of the $\arg F$ gene and that the order of mutants in this linkage group is

$$
\underset{(\text { pyr-2) }}{\text { pyr-3-pyr-I-argF4-arg } F_{3}-\arg F_{1}-\arg F_{5}-\arg F_{2} .}
$$

The two ratio groups indicative of the number of crossovers that occurred ranged between $0-0.04$ and $0.57-1 \cdot 78$.

Table 8. Donor-switching transductions with the $\arg F$ and pyr markers

\begin{tabular}{|c|c|c|c|c|}
\hline \multicolumn{2}{|c|}{ Cross } & \multirow{2}{*}{$\begin{array}{c}\text { Prototroph } \\
\text { transductants }\end{array}$} & \multirow{2}{*}{$\begin{array}{l}\text { Ratio } \\
a / b\end{array}$} & \multirow[b]{2}{*}{ Mutant order } \\
\hline Recipient & Donor & & & \\
\hline $\begin{array}{l}\text { (a) } \arg F_{3} p y r-I \\
\text { (b) pyr-3 }\end{array}$ & $\begin{array}{l}\times \text { pyr }-3 \\
\times \arg F_{3 p y r-I}\end{array}$ & $\begin{array}{r}7 \\
10\end{array}$ & 0.70 & pyr-3 pyr-I arg $F_{3}$ \\
\hline $\begin{array}{l}\text { (a) } \arg F_{3 p y r-I} \\
\text { (b) } \operatorname{argF} 4\end{array}$ & $\begin{array}{l}\times \operatorname{argF} 4 \\
\times \arg F_{3 p y r-I}\end{array}$ & $\begin{array}{r}74 \\
2364\end{array}$ & 0.03 & pyr-I $\arg F_{4} \arg F_{3}$ \\
\hline $\begin{array}{l}\text { (a) } \arg F_{3 p y r-I} \\
\text { (b) } \arg F_{3}\end{array}$ & $\begin{array}{l}\times \arg F_{3} \\
\times \arg F_{3 p y r}-I\end{array}$ & $\begin{array}{l}0 \\
0\end{array}$ & - & - \\
\hline $\begin{array}{l}\text { (a) } \arg F_{3 p y r-I} \\
\text { (b) } \operatorname{argFI}\end{array}$ & $\begin{array}{l}\times \arg F_{I} \\
\times \arg F_{3 p y r-I}\end{array}$ & $\begin{array}{l}4 \\
7\end{array}$ & 0.57 & pyr-I $\arg F_{3} \arg F_{I}$ \\
\hline $\begin{array}{l}\text { (a) } \arg F_{3 p y r-I} \\
\text { (b) argFs }\end{array}$ & $\begin{array}{l}\times \arg F_{5} \\
\times \arg F_{3 p y r}-I\end{array}$ & $\begin{array}{l}10 \\
12\end{array}$ & 0.83 & pyr-1 $\arg F_{3} \arg F_{5}$ \\
\hline $\begin{array}{l}\text { (a) } \arg F_{3 p y r-I} \\
\text { (b) } \operatorname{argFz}\end{array}$ & $\begin{array}{l}\times \operatorname{argF2} \\
\times \operatorname{argF} F_{3 p y r-I}\end{array}$ & $\begin{array}{l}23 \\
13\end{array}$ & $\mathrm{I} \cdot 78$ & pyr-I $\arg F_{3} \operatorname{argF} 2$ \\
\hline $\begin{array}{l}\text { (a) } \arg F_{5 p y r-2} \\
\text { (b) pyr-3 }\end{array}$ & $\begin{array}{l}\times \text { pyr3 } \\
\times \text { argF } 5 \text { pyr-2 }\end{array}$ & $\begin{array}{l}\text { I0 } \\
\text { II }\end{array}$ & 0.91 & pyr-3 pyr2 argFs \\
\hline $\begin{array}{l}\text { (a) } \arg F_{5 p y r-2} \\
\text { (b) } \operatorname{argF} 4\end{array}$ & $\begin{array}{l}\times \operatorname{argF} 4 \\
\times \operatorname{argF} 5 \text { pyr-2 }\end{array}$ & $\begin{array}{r}132 \\
3250\end{array}$ & 0.04 & pyr-2 $\arg F_{4} \arg F_{5}$ \\
\hline $\begin{array}{l}\text { (a) } \arg F_{5 p y r-2} \\
\text { (b) } \arg F_{3}\end{array}$ & $\begin{array}{l}\times \arg F_{3} \\
\times \arg F_{5 p y r-2}\end{array}$ & $\begin{array}{r}0 \\
14\end{array}$ & 0 & pyr-2 $\arg F_{3} \arg F_{5}$ \\
\hline $\begin{array}{l}\text { (a) } \arg F_{5 p y r-2} \\
\text { (b) } \operatorname{argFI}\end{array}$ & $\begin{array}{l}\times \operatorname{argFI} \\
\times \operatorname{argF} 5 \text { pyr-2 }\end{array}$ & $\begin{array}{l}0 \\
5\end{array}$ & 0 & pyr-2 argFI argFs \\
\hline $\begin{array}{l}\text { (a) } \arg F_{5 p y r-2} \\
\text { (b) } \operatorname{argF5}\end{array}$ & $\begin{array}{l}\times \arg F_{5} \\
\times \arg F_{5 p y r-2}\end{array}$ & $\begin{array}{l}\circ \\
\circ\end{array}$ & - & - \\
\hline $\begin{array}{l}\text { (a) } \arg F_{5 p y r-2} \\
\text { (b) } \operatorname{argF2}\end{array}$ & $\begin{array}{l}\times \operatorname{argF} 2 \\
\times \operatorname{argF} 5 \text { pyr-2 }\end{array}$ & $\begin{array}{l}4 \\
5\end{array}$ & 0.80 & $p y r-2 \arg F_{5} \arg F_{2}$ \\
\hline
\end{tabular}

Map of argF sites as determined with the pyr-3 marker. Results of crosses between pyr-3 and the $\arg F$ mutants are given in Table 9. The order determined by donorswitching was confirmed apart from the position of $\operatorname{argF4}$. This mutant behaves like $\operatorname{argH} 2$ mentioned previously and can only be mapped by three-point tests.

Three-point crosses between $\arg D$ and str-r markers. Double mutants with five known $\arg D$ and $s t r-r$ markers (Table I) were crossed with five single $\arg D$ mutants in donor-switching experiments (Table 10). Selection was made for $\arg ^{+}$str-r transductants. Two ratio classes $(0-0 \cdot 3$ and $1 \cdot 3-2 \cdot 3)$ were again identified. Results indicate that the str-r mutations are on the same side of the $\arg D$ gene and that the mutant order within this linkage group is $s t r-r-\arg D_{5}-\arg D_{2}-\arg D_{1-\arg D 6-\arg D_{3}}$. 
Relative distances in the argD-str-r linkage group. Results of a ratio test with str-rI as donor and $\arg D$ mutants as recipients are given in Table I I. Mutant $\arg D_{4}$, which could not be mapped previously because of its poor recipient capacity (Table 3), could now be placed. These results confirm the order derived from donor-switching experiments excepting the position of $\operatorname{argD2}$. This mutant behaves like $\operatorname{argHz}$ and $\operatorname{argF} 4$. The map of this linkage group is presented in Fig. 2.

Table 9. Distances between argF mutants and the pyr-3 marker

\begin{tabular}{|c|c|c|c|}
\hline \multicolumn{4}{|c|}{ Values are the mean of two experiments. } \\
\hline Recipient & $\begin{array}{l}\text { Transductants: } \\
\text { prototroph/total }\end{array}$ & $\%$ & Average \\
\hline $\begin{array}{l}\operatorname{argF} 4 \times \text { pyr-3 } \\
\text { pyr-3 } \times \operatorname{argF} 4\end{array}$ & $\begin{array}{l}6600 / 12900 \\
2277 / 5870\end{array}$ & $\left.\begin{array}{l}5 I \cdot 2 \\
38 \cdot 8\end{array}\right\}$ & $45^{\circ} 0$ \\
\hline $\begin{array}{l}\text { arg } F_{3} \times p y r-3 \\
\text { pyr-3 } \times \text { arg } F_{3}\end{array}$ & $\begin{array}{l}52 / 2464 \\
38 / 2208\end{array}$ & $\left.\begin{array}{l}2 \cdot I \\
I \cdot 8\end{array}\right\}$ & $2 \cdot 0$ \\
\hline $\begin{array}{l}\arg F I \times p y r-3 \\
p y r-3 \times \arg F I\end{array}$ & $\begin{array}{l}62 / 2142 \\
46 / 1988\end{array}$ & $\left.\begin{array}{l}2 \cdot 9 \\
2 \cdot 3\end{array}\right\}$ & $2 \cdot 6$ \\
\hline $\begin{array}{l}\arg F_{5} \times p y r-3 \\
p y r-3 \times \arg F_{5}\end{array}$ & $\begin{array}{l}193 / 5116 \\
101 / 2965\end{array}$ & $\left.\begin{array}{l}3 \cdot 8 \\
3 \cdot 4\end{array}\right\}$ & $3 \cdot 6$ \\
\hline $\begin{array}{l}\operatorname{argF2} \times \text { pyr-3 } \\
\text { pyr-5 } \times \text { argF } 2\end{array}$ & $\begin{array}{l}101 / 2035 \\
103 / 2242\end{array}$ & $\left.\begin{array}{l}5 \cdot 0 \\
4 \cdot 6\end{array}\right\}$ & $4 \cdot 8$ \\
\hline
\end{tabular}

Order of $\arg A$ sites as determined with the non-linked str-r marker. Results of a typical experiment are given in Table 12. Mutants $\arg A I$ and $\arg A 4$ were derived from str-r-I and could only be used as donors in these experiments. Results obtained were used to construct a 'best-fit' map (Hartman et al. 1960), which is presented in Fig. 2.

The arginine gene maps of Proteus mirabilis, Escherichia coli and Salmonella typhimurium. These are presented in Fig. 3. The clustering of $\arg E, C, B, H$ in that order ( $E$. coli K-I2), the linkage of $\arg D$ to $\operatorname{str}-r(E$. coli w) and the non-linkage of $\arg F$ and $\arg A$ to the other arginine genes are common characteristics. The $\arg G$ gene is included in the $\arg E, C, B, G, H$ group in $P$. mirabilis and this cluster is closely linked to cys $E$ not to met genes as in the other organisms. The $\arg F$ gene is linked to a $p y r$ marker in $P$. mirabilis like the additional gene for the same enzymic step $\arg I$ in $E$. coli $\mathrm{K}-12$ (Glansdorff, Sand \& Verhoef, I967; Taylor \& Thoman, 1964).

\section{DISCUSSION}

Double mutants constructed by transduction (Glansdorff, 1965) would have been preferable to the selected double mutants used here for the reason that the second mutations in selected double mutants cannot be used in simple ratio tests because they are not available separately. Phage 34/13 causes lysogenic conversion (Coetzee, 196I) which results in non-adsorption of homologous phage. Despite the use of low multiplicities of infection and antiserum to phage 34/13 (Coetzee \& Sacks, 1960b) transductants were invariably lysogenized and could not be re-transduced. Attempts to cure the transductants (Zinder, 1958) were also unsuccessful (Prozesky, unpublished results).

Abortive transduction with the system used here has not been reported (Coetzee \& Sacks, I960a; Coetzee, de Klerk \& Maré, 1963; Coetzee, 1963). Minute colonies 
Table 10. Three-point transductions with the $\arg D$ and str-r markers*

\begin{tabular}{|c|c|c|c|c|}
\hline \multicolumn{2}{|l|}{ Cross } & \multirow{2}{*}{$\begin{array}{c}\text { Transductants } \\
\text { arg }^{+}, s t r-r\end{array}$} & \multirow{2}{*}{$\begin{array}{c}\text { Ratio } \\
a / b\end{array}$} & \multirow[b]{2}{*}{ Mutant order } \\
\hline Recipient & Donor & & & \\
\hline (a) $\arg D_{I}$ & $\times$ argDsstr-r-ro & 0 & 0 & str-r-10 $\arg D_{5} \arg D_{r}$ \\
\hline (b) argDsstr-r-Io & $\times \arg D r$ & 14 & & \\
\hline $\begin{array}{l}\text { (a) } \arg D_{2} \\
\text { (b) } \arg D_{5 \text { str-r-1o }}\end{array}$ & $\begin{array}{l}\times \arg D_{5 s t r-r-10} \\
\times \arg D_{2}\end{array}$ & $\begin{array}{l}0 \\
9\end{array}$ & 0 & str-r-10 $\arg D_{5} \arg D_{2}$ \\
\hline $\begin{array}{l}\text { (a) } \arg D_{3} \\
\text { (b) } \arg D_{5 s t r-r-10}\end{array}$ & $\begin{array}{l}\times \arg D_{5 s t r-r-10} \\
\times \arg D_{3}\end{array}$ & $\begin{array}{r}3 \\
21\end{array}$ & $0 \cdot I$ & str-r-1o $\arg D_{5} \arg D_{3}$ \\
\hline $\begin{array}{l}\text { (a) } \arg D_{5} \\
\text { (b) } \arg D_{5} \text { str-r-1o }\end{array}$ & $\begin{array}{l}\times \arg D_{I s t r-5 r-10} \\
\times \arg D_{5}\end{array}$ & $\begin{array}{l}0 \\
0\end{array}$ & - & 一 \\
\hline $\begin{array}{l}\text { (a) } \operatorname{argD6} \\
\text { (b) } \arg D_{5 s t r-r-10}\end{array}$ & $\begin{array}{l}\times \arg D_{5 s t r-r-10} \\
\times \operatorname{argD6}\end{array}$ & $\begin{array}{r}2 \\
13\end{array}$ & 0.2 & str-r-10 $\arg D_{5} \arg D 6$ \\
\hline $\begin{array}{l}\text { (a) } \operatorname{argDI} \\
\text { (b) } \arg D_{2 s t r-r-7}\end{array}$ & $\begin{array}{l}\times \arg D_{2 s t r-r-7} \\
\times \arg D_{I}\end{array}$ & $\begin{array}{r}3 \\
13\end{array}$ & 0.2 & str-r-7 $\arg D_{2} \arg D_{1}$ \\
\hline $\begin{array}{l}\text { (a) } \operatorname{argD2} \\
\text { (b) } \operatorname{argD2str-r-7}\end{array}$ & $\begin{array}{l}\times \operatorname{argD} 2 s t r-r-7 \\
\times \arg D_{2}\end{array}$ & $\begin{array}{l}0 \\
0\end{array}$ & - & - \\
\hline $\begin{array}{l}\text { (a) } \arg D_{3} \\
\text { (b) } \arg D 2 s t r-r-7\end{array}$ & $\begin{array}{l}\times \operatorname{argD2str-r-7} \\
\times \arg D_{3}\end{array}$ & $\begin{array}{r}0 \\
17\end{array}$ & 0 & str-r-7 $\arg D_{2} \arg D_{3}$ \\
\hline $\begin{array}{l}\text { (a) } \arg D_{5} \\
\text { (b) argD2str-r-7 }\end{array}$ & $\begin{array}{l}\times \arg D_{2 s t r-r-7} \\
\times \arg D_{5}\end{array}$ & $\begin{array}{l}17 \\
\text { I I }\end{array}$ & $1 \cdot 6$ & str-r-7 $\arg D_{5} \arg D_{2}$ \\
\hline $\begin{array}{l}\text { (a) } \operatorname{argD6} \\
\text { (b) } \operatorname{argD2str-r-7}\end{array}$ & $\begin{array}{l}\times \text { argD2str-r-7 } \\
\times \text { argD6 }\end{array}$ & $\begin{array}{r}5 \\
20\end{array}$ & 0.3 & str-r-7 arg D2 argD6 \\
\hline $\begin{array}{l}\text { (a) } \arg D I \\
\text { (b) } \arg D 1 s t r-r-6\end{array}$ & $\begin{array}{l}\times \arg D_{1 s t r-r-6} \\
\times \arg D_{I}\end{array}$ & $\begin{array}{l}0 \\
0\end{array}$ & - & - \\
\hline $\begin{array}{l}\text { (a) } \arg D_{2} \\
\text { (b) } \arg D_{1 s t r-r-6}\end{array}$ & $\begin{array}{l}\times \arg D_{1 s t r-r-6} \\
\times \operatorname{argD} D_{2}\end{array}$ & $\begin{array}{l}7 \\
5\end{array}$ & $1 \cdot 4$ & str-r-6 $\arg D_{2} \arg D_{r}$ \\
\hline $\begin{array}{l}\text { (a) } \arg D_{3} \\
\text { (b) } \arg D_{1 s t r-r-6}\end{array}$ & $\begin{array}{l}\times \arg D_{1 s t r-r-6} \\
\times \arg D_{3}\end{array}$ & $\begin{array}{r}4 \\
22\end{array}$ & 0.2 & str-r-6 $\arg D_{1} \arg D_{3}$ \\
\hline $\begin{array}{l}\text { (a) } \operatorname{argD6} \\
\text { (b) } \operatorname{argDrstr-r-6}\end{array}$ & $\begin{array}{l}\times \operatorname{argDIstr-r-6} \\
\times \operatorname{argD6}\end{array}$ & $\begin{array}{l}0 \\
6\end{array}$ & 0 & str-r-6 argDI argD6 \\
\hline $\begin{array}{l}\text { (a) } \operatorname{argDI} \\
\text { (b) } \arg D 6 s t r-r-I I\end{array}$ & $\begin{array}{l}\times \operatorname{argD6} \text { str-r-II } \\
\times \arg D_{I}\end{array}$ & $\begin{array}{l}6 \\
4\end{array}$ & $1 \cdot 5$ & str-r-II $\arg D I$ argD6 \\
\hline $\begin{array}{l}\text { (a) } \arg D_{3} \\
\text { (b) } \arg D 6 s t r-r-I I\end{array}$ & $\begin{array}{l}\times \operatorname{argD6str-r-II} \\
\times \arg D_{3}\end{array}$ & $\begin{array}{l}1 \\
5\end{array}$ & 0.2 & str-r-II $\arg D_{6} \arg D_{3}$ \\
\hline $\begin{array}{l}\text { (a) } \arg D_{5} \\
\text { (b) } \arg D 6 s t r-r-I I\end{array}$ & $\begin{array}{l}\times \operatorname{argD6str-r-II} \\
\times \arg D_{5}\end{array}$ & $\begin{array}{l}4 \\
3\end{array}$ & $1 \cdot 3$ & str-r-II $\arg D_{5}$ argD6 \\
\hline $\begin{array}{l}\text { (a) } \operatorname{argD6} \\
\text { (b) } \operatorname{argD6str-r-1I}\end{array}$ & $\begin{array}{l}\times \operatorname{argD6} \text { str-r-II } \\
\times \arg D 6\end{array}$ & $\begin{array}{l}0 \\
0\end{array}$ & - & 一 \\
\hline $\begin{array}{l}\text { (a) } \arg D_{I} \\
\text { (b) } \arg D_{3 s t r-r-8}\end{array}$ & $\begin{array}{l}\times \arg D_{3 s t r-r-8} \\
\times \arg D_{I}\end{array}$ & $\begin{array}{l}72 \\
40\end{array}$ & $1 \cdot 8$ & str-r-8 $\arg D_{1} \arg D_{3}$ \\
\hline $\begin{array}{l}\text { (a) } \arg D_{2} \\
\text { (b) } \arg D_{3 s t r-r-8}\end{array}$ & $\begin{array}{l}\times \arg D_{3 s t r-r-8} \\
\times \operatorname{argD2}\end{array}$ & $\begin{array}{c}10 \\
8\end{array}$ & $1 \cdot 3$ & str-r-8 $\arg D_{2} \arg D_{3}$ \\
\hline $\begin{array}{l}\text { (a) } \arg D_{3} \\
\text { (b) } \arg D_{3 s t r-r-8}\end{array}$ & $\begin{array}{l}\times \arg D_{3 s t r-r-8} \\
\times \arg D_{3}\end{array}$ & $\begin{array}{l}0 \\
0\end{array}$ & - & - \\
\hline $\begin{array}{l}\text { (a) } \arg D_{5} \\
\text { (b) } \arg D_{3 s t r-r-8}\end{array}$ & $\begin{array}{l}\times \arg D_{3 s t r-r}-8 \\
\times \arg D_{5}\end{array}$ & $\begin{array}{l}82 \\
41\end{array}$ & $2 \cdot 0$ & str-r-8 $\arg D_{5} \arg D_{3}$ \\
\hline $\begin{array}{l}\text { (a) } \arg D_{6} \\
\text { (b) } \arg D_{3 s t r-r-8}\end{array}$ & $\begin{array}{l}\times \arg D_{3 s t r-r}-8 \\
\times \operatorname{argD6}\end{array}$ & $\begin{array}{l}46 \\
20\end{array}$ & $2 \cdot 3$ & str-r-8 $\arg D_{6} \arg D_{3}$ \\
\hline
\end{tabular}

* $\arg \mathrm{D}_{4}$ was not used in this investigation as it is a poor recipient with average donor capacity. 
resembling abortive transductant colonies were sometimes observed in this investigation but attempts to prove them so (Ozeki, 1956; Nishioka, Demerec \& Eisenstark, 1967) did not yield reproducible results and this problem is being investigated further.

Proteus mirabilis, Escherichia coli and Salmonella typhimurium have guanine + cytosine molar contents of 38-41 \%, 50\% (Hill, 1966) and 50-52\% (Marmur, Falkow \& Mandel, 1963) respectively. Notable genetic differences between $P$. mirabilis and the other two organisms were expected. The different linkage of the $\arg E, C, B, G, H$ cluster found here may mean that the placing of this cluster differs in $P$. mirabilis and $E$. coli. However, we are looking at the genetic map of Proteus through the keyhole of

Table I I. Distances between $\arg D$ mutants and the str-r-I marker

Values are the mean of two experiments

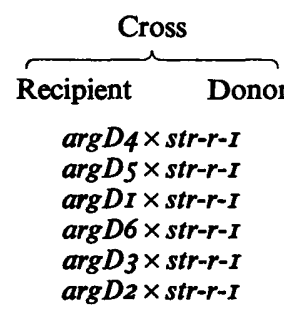

$\begin{array}{cc}\text { arg }^{+}, \text {str-r } & \text { str-r } \\ 108 & 1208 \\ 185 & 2355 \\ 447 & 5719 \\ 310 & 5132 \\ 411 & 5828 \\ 134 & 2628\end{array}$

$\begin{array}{cc}\text { Ratio } & \\ \text { str-r-arg }{ }^{+}, \text {str-r } / \text { str-r } & \% \\ 1100 / 1208 & 91 \cdot 2 \\ 2170 / 2355 & 92 \cdot 1 \\ 5272 / 5719 & 92 \cdot 2 \\ 4822 / 5132 & 94 \cdot 0 \\ 5417 / 5828 & 94 \cdot 7 \\ 2494 / 2628 & 94 \cdot 9\end{array}$

Table 12. Mapping of the argA mutants with prototroph transductant numbers normalized with respect to str-r marker transduction in the same crosses

\begin{tabular}{|c|c|c|c|c|}
\hline \multirow[b]{2}{*}{ Recipient* } & \multicolumn{2}{|c|}{ No. transductants } & \multirow{2}{*}{$\begin{array}{c}\text { Ratio } \\
\arg ^{+} / \text {str-r } \%\end{array}$} & \multirow[b]{2}{*}{ Average } \\
\hline & Prototroph & str-r & & \\
\hline $\arg A 2 \times \arg A I s t r-r-I$ & 2 & 484 & 0.41 & \\
\hline $\arg A_{3} \times \arg A$ Istr-r-I & 36 & 543 & 6.63 & \\
\hline $\arg A 5 \times \arg A I s t r-r-1$ & 59 & 915 & 6.45 & \\
\hline $\arg A 6 \times \arg A 1 s t r-r-1$ & 47 & 851 & 3.53 & \\
\hline $\arg A 2 \times \arg A 4$ str-r-I & 4 & 139 & $2 \cdot 88$ & \\
\hline $\arg A_{3} \times \arg A_{4}$ str-r-I & 12 & 285 & $4 \cdot 21$ & \\
\hline $\arg A 5 \times \arg A 4$ str-r-I & 8 & 227 & 3.52 & \\
\hline $\arg A 6 \times \arg A 4$ str-r-I & $\mathbf{I}$ & 198 & 0.51 & \\
\hline $\arg A 2 \times \arg A 2 s t r-r-2$ & 0 & 186 & - & \\
\hline $\begin{array}{l}\arg A_{3} \times \arg A 2 s t r-r-2 \\
\arg A 2 \times \arg A 3 s t r-r-3\end{array}$ & $\begin{array}{r}20 \\
8\end{array}$ & $\begin{array}{l}306 \\
126\end{array}$ & $\left.\begin{array}{l}6 \cdot 54 \\
6 \cdot 35\end{array}\right\}$ & $6 \cdot 45$ \\
\hline $\begin{array}{l}\arg A 2 \times \arg A 5 s t r-r-4 \\
\arg A 5 \times \arg A 2 s t r-r-2\end{array}$ & $\begin{array}{r}6 \\
23\end{array}$ & $\begin{array}{l}138 \\
312\end{array}$ & $\left.\begin{array}{l}4 \cdot 35 \\
7 \cdot 37\end{array}\right\}$ & $5 \cdot 86$ \\
\hline & & & 831 & \\
\hline $\begin{array}{l}\arg A 2 \times \arg A 6 s t r-r-5 \\
\arg A 6 \times \arg A 2 s t r-r-2\end{array}$ & $\begin{array}{r}9 \\
16\end{array}$ & $\begin{array}{l}165 \\
298\end{array}$ & $\left.\begin{array}{l}5 \cdot 45 \\
5 \cdot 37\end{array}\right\}$ & $5 \cdot 41$ \\
\hline $\arg A_{3} \times \arg A_{3} s t r-r-3$ & 0 & 288 & - & \\
\hline $\begin{array}{l}\arg A_{5} \times \arg A_{3 s t r-r-3} \\
\arg A_{3} \times \arg A_{5 s t r-r-4}\end{array}$ & $\begin{array}{l}2 \\
5\end{array}$ & $\begin{array}{l}207 \\
289\end{array}$ & $\left.\begin{array}{l}0.97 \\
1 \cdot 73\end{array}\right\}$ & $1 \cdot 35$ \\
\hline $\begin{array}{l}\arg A 6 \times \arg A 3 s t r-r-3 \\
\arg A_{3} \times \arg A 6 s t r-r-5\end{array}$ & $\begin{array}{l}4 \\
3\end{array}$ & $\begin{array}{l}203 \\
294\end{array}$ & $\left.\begin{array}{l}1 \cdot 97 \\
1 \cdot 02\end{array}\right\}$ & $I \cdot 50$ \\
\hline $\arg A 5 \times \arg A 6 s t r-r-5$ & $\mathbf{I}$ & 286 & $0.35\}$ & 0.43 \\
\hline $\arg A 6 \times \arg A 5 s t r-r-4$ & $\mathbf{I}$ & 197 & $0.51\}$ & \\
\hline $\arg A 6 \times \arg A 6 s t r-r-5$ & 0 & 226 & - & \\
\hline
\end{tabular}


transduction and no firm conclusions as to the orientation of the linkage groups can be reached. The spatial arrangements of the arginine genes themselves are similar in $P$. mirabilis, $E$. coli and $S$. typhimurium. This may be necessary for the function and regulation of these genes and could be an evolutionary trait. Mutants with defective control of arginine synthesis have been isolated in P. mirabilis strain 13 (Prozesky, in

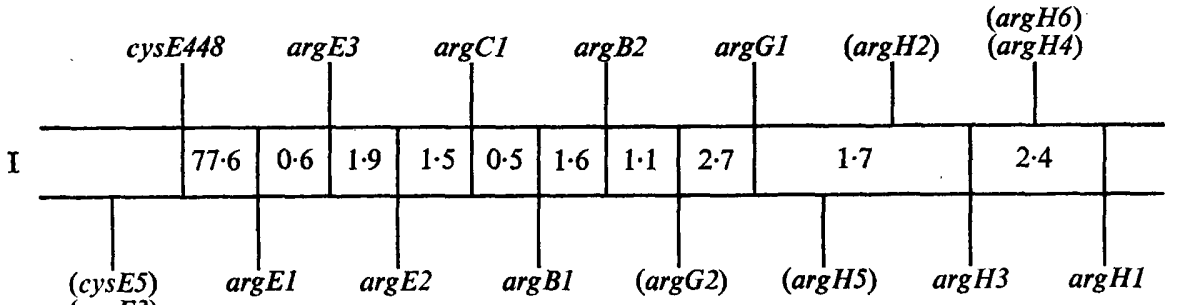

(cysE3)

II

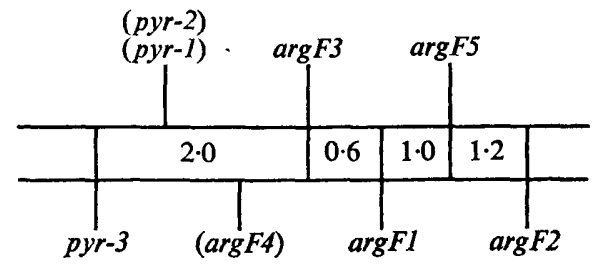

III

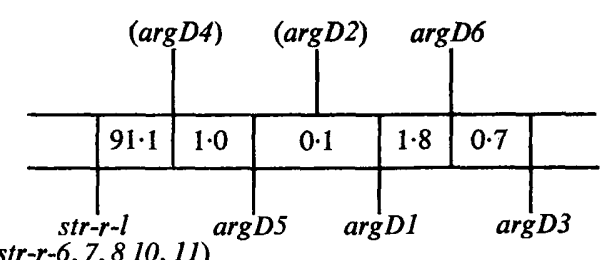

IV

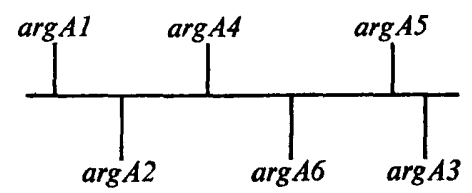

Fig. 2. Map of mutations in the four linkage groups. Mutants in brackets were not mapped by all methods or gave inconclusive results. The tentative positions assigned to such mutants are based on results obtained from donor-switching experiments except for mutant argGz, where results of the ratio test were used. The relative distances in linkage group I are those estimated with cysE 448 as the linked reference marker. In linkage group IV only the order of mutants was established and relative distances are tentative.

preparation) and it will be of interest to know whether the control of the $\arg G$ gene in $P$. mirabilis and $E$. coli differs. In $E$. coli this gene is not included in the $\arg E, C, B, H$ cluster and is distantly linked by transduction to the regulator gene $\arg R$ (Jacoby \& Gorini, 1967). It may also be of taxonomic interest to know whether other members of the Proteus-Providence group have the same arrangement and linkage relationships of their arginine genes as P. mirabilis (Coetzee, Smit \& Prozesky, 1966). 
Escherichia coli $\mathrm{K}-12$

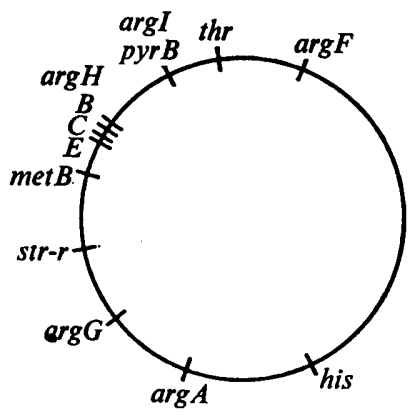

Salmonella typhimurium

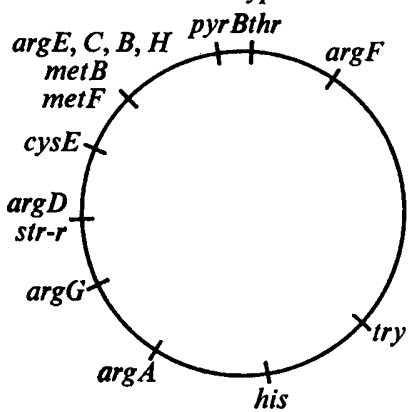

Escherichia coll $\mathbb{W}$

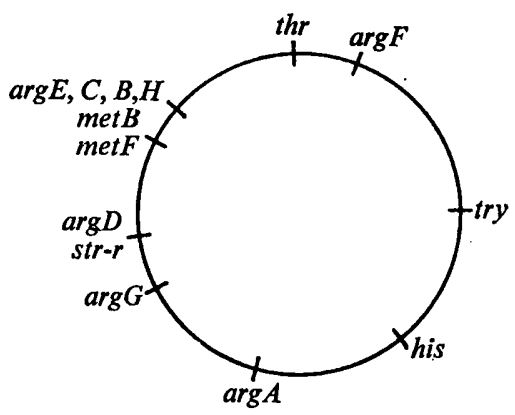

Proteus mirabilis 13

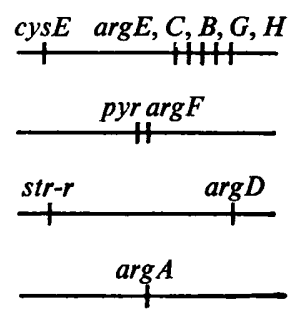

Fig. 3. The arrangement of arg genes in the linkage maps of Escherichia coli strains K-12 and $\mathrm{W}$ and Salmonella typhimurium compared to the arrangement of these genes in the four linkage groups mapped by transduction in Proteus mirabilis strain 13. The orientation of the Proteus mirabilis linkage groups and their positions relative to one another are unknown. The Escherichia coli maps are given according to Glansdorff, Sand \& Verhoef (1967) and Vogel \& Bacon (1966) for strains K-12 and w respectively. The map for Salmonella typhimurium is that of Sanderson \& Demerec (1965). Maps are not drawn to scale. arg = arginineless; $s t r-r=$ streptomycin-resistant $; c y s=$ cysteineless; $p y r=$ pyrimidineless; try $=$ tryptophanless; his $=$ histidineless; met $=$ methionineless; $t h r=$ threonineless.

This work was supported by grants from the South African Council for Scientific and Industrial Research to Professor J. N. Coetzee.

\section{REFERENCES}

Adams, M. H. (1959). In Bacteriophages. New York: Interscience Publishers Inc.

ARMSTRONG, F. B. (1967). Orientation and order of the met-arg region in the Salmonella typhimurium linkage map. Genetics 56, 463.

Baumberg, S., BaCon, D. F. \& Vogel, H. J. (1965). Individually repressible enzymes specified by clustered genes of arginine synthesis. Proc. natn. Acad. Sci. U.S.A. 53, 1029.

Baumberg, S., Bacon, D. F. \& VoGel, H. J. (1966). A mutation affecting the repression-derepression behaviour of three out of four enzymes specified by clustered genes of arginine synthesis in Escherichia coli. Genetics 54, 322.

Clowes, R. C. (1960). Fine genetic structure as revealed by transduction. Symp. Soc. gen. Microbiol. IO, 92. 
Coetzee, J. N. (1961). Lysogenic conversion in the genus Proteus. Nature, Lond. x89, 946.

Coetzee, J. N. (1963). Transduction of swarming in Proteus mirabilis. J. gen. Microbiol. 33, I.

CoetzeE, J. N. \& SACKs, T. G. (I960a). Morphological variants of Proteus hauseri. J. gen. Microbiol. 23, 209.

Coetzee, J. N. \& SaCks, T. G. (1960 b). Transduction of streptomycin resistance in Proteus mirabilis. J. gen. Microbiol. 23, 445 .

Coetzee, J. N., DE KLERK, H. C. \& MARÉ, I. J. (1963). Sodium azide resistance in Proteus hauseri. J. gen. Microbiol. 33, 313.

Coetzee, J. N., Smit, J. A. \& Prozesky, O. W. (1966). Properties of Providence and Proteus morganii transducing phages. J. gen. Microbiol. 44, 167.

Demerec, M., Moser, H., Clowes, R. C., Lahr, E. L., Ozeki, H. \& Vielmetter, W. (I956). Genetic studies with bacteria. Yb. Carnegie Instn Wash. 55, 30I.

Demerec, M., Lahr, E. L., Balbinder, E., Miyake, T., Ishidsu, J., Mizobuchi, K. \& Mahler, B. (1960). Bacterial genetics. Yb. Carnegie Instn Wash. 59, 426.

GlaNSDORFF, N. (1965). Topography of cotransducible arginine mutations in Escherichia coli K-I 2. Genetics 51, 167.

GlansdorfF, N. (1967). Pseudoinversions in the chromosome of Escherichia coli K-1 2. Genetics 55 , 49.

GLANSDORFF, N., SAND, G. \& VERHOEF, C. (1967). The dual genetic control of ornithine transcarbamylase synthesis in Escherichia coli $\mathrm{K}-\mathrm{I}$ 2. Mut. Res. 4, 743.

Grabow, W. O. K. \& SMT, J. A. (1967). Methionine synthesis in Proteus mirabilis. J. gen. Microbiol. 46, 47.

Gross, J. D. \& ENGlesberg, E. (1959). Determination of the order of mutational sites governing $\mathrm{L}$-arabinose utilisation in Escherichia coli $\mathrm{B} / \mathrm{r}$ by transduction with phage Plbt. Virology 9, 314.

Hartman, P. E., Loper, J. C. \& Serman, D. (I960). Fine structure mapping by complete transduction between histidine-requiring Salmonella mutants. J. gen. Microbiol. 22, 323.

Hill, L. R. (1966). An index to deoxyribonucleic acid base compositions of bacterial species. J. gen. Microbiol. 44, 419.

Jacob, F., Perrin, D., Sanchez, C. \& Monod, J. (1960). L'opéron: groupe de gènes à expression coordonneé par un opérateur. C. r. hebd. Séanc. Acad. Sci., Paris 250, 1727.

JACOBY, G. A. \& GoRINI, L. (1967). Genetics of control of the arginine pathway in Escherichia coli B and K. J. molec. Biol. 24, $4 \mathrm{I}$.

LEDERBERG, J. \& LeDERBERG, E. M. (1952). Replica plating and indirect selection of bacterial mutants. J. Bact. 63, 399.

LENNOX, E. S. (1955). Transduction of linked genetic characters of the host by bacteriophage Pl. Virology I, 190.

MAAs, W. K. (1961). Studies on repression of arginine biosynthesis in Escherichia coli. Cold Spring Harb. Symp. quant. Biol. 26, 183.

MAAs, R. \& MAAs, W. K. (1962). Introduction of a gene from Escherichia coli в into Hfr and F- strains of Escherichia coli K-12. Proc. natn. Acad. Sci. U.S.A. 48, 1887.

Marmur, J., Falkow, S. \& Mandel, M. (I963). New approaches to bacterial taxonomy. A. Rev. Microbiol. I7, 329.

Masters, M. \& PARDeE, A. B. (1965). Sequence of enzyme synthesis and gene replication during the cell cycle of Bacillus subtilis. Proc. natn. Acad. Sci. U.S.A. 48, 1887.

NishiokA, Y., Demerec, M. \& EISENSTARK, A. (I967). Genetic analysis of aromatic mutants of Salmonella typhimurium. Genetics 56, 34I.

OzEKI, H. (1956). Abortive transduction in purine-requiring mutants of Salmonella typhimurium. In Genetic Studies with Bacteria. Publs Carnegie Instn. 612, 97.

Pritchard, R. H. (1960). Localised negative interference and its bearing on models of gene recombination. Genet. Res., Camb. I, I.

Prozesky, O. W. (1967). Arginine synthesis in Proteus mirabilis. J. gen. Microbiol. 49, 325.

Prozesky, O. W. \& Coetzee, J. N. (I966). Linked transduction in Proteus mirabilis. Nature, Lond. 209, 1262 .

Prozesky, O. W., De KLeRK, H. C. \& Coetzee, J. N. (1965). The morphology of Proteus bacteriophages. J. gen. Microbiol. 41, 29.

SAND, G. \& Glansdorff, N. (1967). L'opéron arginine d'Escherichia coli. Archs int. Physiol. Biochim. 75,568 .

Sanderson, K. E. \& Demerec, M. (1965). The linkage map of Salmonella typhimurium. Genetics 5I, 897. 
TAYLOR, A. L. \& THOMAN, M. S. (1964). The genetic map of Escherichia coli K-12. Genetics 50, 659.

VOGEL, H. J. \& BACON, D. F. (I966). Gene aggregation: Evidence for a coming together of functionally related not closely linked genes. Proc. natn. Acad. Sci. U.S.A. 55, 1456.

VOGEL, H. J., BACON, D. F. \& BAICH, A. (1963). Induction of acetyl-ornithine- $d$-transaminase during pathway-wide repression. In Informational Macromolecules, p 293. Ed. by H. J. Vogel, V. Bryson and J. O. Lampen. New York: Academic Press.

ZINDER, N. D. (1958). Lysogenisation and super-infection immunity in Salmonella. Virology 5, 291. 